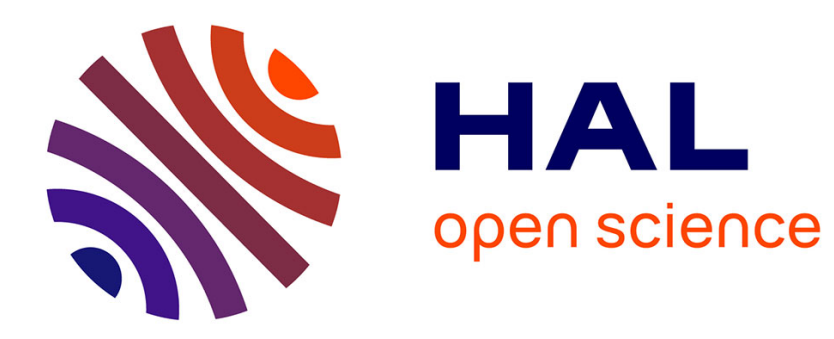

\title{
Log-likelihood ratio test for detecting transient change
}

Daniela Jarušková, Vladimir I. Piterbarg

\section{To cite this version:}

Daniela Jarušková, Vladimir I. Piterbarg. Log-likelihood ratio test for detecting transient change. Statistics and Probability Letters, 2011, 81 (5), pp.552. 10.1016/j.spl.2011.01.006 . hal-00725105

\section{HAL Id: hal-00725105 \\ https://hal.science/hal-00725105}

Submitted on 24 Aug 2012

HAL is a multi-disciplinary open access archive for the deposit and dissemination of scientific research documents, whether they are published or not. The documents may come from teaching and research institutions in France or abroad, or from public or private research centers.
L'archive ouverte pluridisciplinaire HAL, est destinée au dépôt et à la diffusion de documents scientifiques de niveau recherche, publiés ou non, émanant des établissements d'enseignement et de recherche français ou étrangers, des laboratoires publics ou privés. 


\section{Accepted Manuscript}

Log - likelihood ratio test for detecting transient change

Daniela Jarušková, Vladimir I. Piterbarg

PII:

$$
\text { S0167-7152(11)00013-7 }
$$

DOI: 10.1016/j.spl.2011.01.006

Reference: STAPRO 5881

To appear in: $\quad$ Statistics and Probability Letters

Received date: 5 October 2009

Revised date: 11 January 2011

Accepted date: 11 January 2011

Please cite this article as: Jarušková, D., Piterbarg, V.I., Log - likelihood ratio test for detecting transient change. Statistics and Probability Letters (2011), doi:10.1016/j.spl.2011.01.006

This is a PDF file of an unedited manuscript that has been accepted for publication. As a service to our customers we are providing this early version of the manuscript. The manuscript will undergo copyediting, typesetting, and review of the resulting proof before it is published in its final form. Please note that during the production process errors may be discovered which could affect the content, and all legal disclaimers that apply to the journal pertain. 


\title{
Log-likelihood ratio test for detecting transient change
}

\author{
Daniela Jarušková*, Vladimir I. Piterbarg \\ Department of Mathematics, Czech Technical University, Thákurova 7, CZ 16629 \\ Praha 6, Czech Republic \\ Faculty of Mathematics and Mechanics, Laboratory of Probability, Moscow State \\ University, 119899 Moscow, Russia
}

\begin{abstract}
The limit behaviour of scan statistics in a form of maxima of moving sum processes with changing window size for detecting a transient change is studied. Approximate critical values obtained from tail behaviour of the limit variables that are maxima of some locally homogeneous Gaussian fields are compared with critical values obtained by simulation.
\end{abstract}

Keywords: change points detection, scan statistics, asymptotics, maxima of locally homogeneous Gaussian fields

\section{Introduction}

In many situations we observe at equidistant time points a sequence of random vectors $\left\{\boldsymbol{X}_{i}, i=1, \ldots, n\right\}$, or on a grid in a square $\{(i, j), i=$ $1, \ldots, n ; j=1, \ldots, n\}$ an array of vectors $\left\{\boldsymbol{X}_{i j}, i=1, \ldots, n ; j=1, \ldots, n\right\}$. The question may arise whether all observed vectors have the same mean or whether for some $i \in\left[t_{1}, t_{2}\right]$, resp. for $(i, j) \in A \subset\left[x_{1}, x_{2}\right] \times\left[y_{1}, y_{2}\right]$, at least one coordinate of the mean vector is different. For example, components of vectors $\left\{\boldsymbol{X}_{i}\right\}$ may represent signals that are transmitted through several channels and we are interested to detect the transient change in signal values. In medicine the one-dimensional variables $\left\{X_{i}\right\}$ may represent numbers per day of new cases of a disease and an increase of their mean

\footnotetext{
${ }^{*}$ Corresponding author

Email addresses: jarus@mat.fsv.cvut.cz (Daniela Jarušková), piter@mech.math.msu.su (Vladimir I. Piterbarg) 
value for a certain time period means an epidemic outbreak. In the control of a homogeneity of a textile fabric the variables $\left\{X_{i j}\right\}$ may represent values of a pixel with the coordinates $(i, j)$ in a digital black and white photo. The goal is to detect a speck (a region of a fabric) where a mean gray level is different. The useful tools in statistical inference are scan statistics based on moving sum process $\left\{\boldsymbol{S}([t, t+a])=\sum_{i=t}^{t+a}\left(\boldsymbol{X}_{i}-\overline{\boldsymbol{X}}\right), t=1, \ldots, n-a\right\}$, resp. $\left\{\boldsymbol{S}([t, t+a] \times[s, s+b])=\sum_{i=t}^{t+a} \sum_{j=s}^{s+b}\left(\boldsymbol{X}_{i j}-\overline{\boldsymbol{X}}\right), t=1, \ldots, n-a ; s=\right.$ $1, \ldots, n-b\}$, where $\overline{\boldsymbol{X}}$ is the overall mean, for more details see Glaz et al. (2001). Sometimes, it is difficult to say how long an epidemic may last or how large a speck of a different grey level may be. In such a case the window width is not specified in advance but scan statistics with varying window width are considered. This corresponds to the situation studied in our paper.

The problem may be generalized to a search for a transient change in parameters of a regular distribution or for a transient change in an intensity of a Poisson process.

In the scope of mathematical statistics a decision whether parameters of interest changed is based on hypotheses testing where the null hypothesis claims that there is no change while the alternative claims that during some time interval or in some place the observed variables or vectors are distributed according to a distribution with different parameters. The described problem belongs to the change point analysis and maximum type test statistics based on standardized partial moving sum process may be applied. For applying them we need to know corresponding critical values. As an exact distribution under the null hypothesis is very complex we usually apply an asymptotic distribution. As always, the maximum type test statistics tend to infinity and to get a limit distribution we have to consider their "trimmed versions". In such a case the limit variables correspond to maxima of Gaussian fields on compact sets.

The exact distributions of limit variables are unknown, but approximate critical values may be obtained from their tail behaviour. The exceedance probability of Gaussian fields of this type (as the exceedance level tends to infinity) was studied by Siegmund (1988) and Siegmund and Yakir (2000). Yao (1993) obtained an approximation for detecting a transient change in mean of normally distributed random variables while Loader (1991) obtained an approximation for a special case of detecting a transient change in an intensity of a one and two-dimensional Poisson process. We obtained tail approximations of the limit variables by the double-sum method for locally homoge- 
neous Gaussian fields suggested by Piterbarg (1996). More precisely, we have shown that the conditions of Theorem 7.1, Chapter 2 of Piterbarg (1996) are satisfied. A recently published paper by Chan and Lai (2006) presents similar ideas of tail approximation for nonhomogeneous Gaussian fields. As a consequence of their Theorem 2.1, see Example 2.2, they obtained a highlevel exceedance probability for a maximum of standardized increments of a Wiener process. It is clear that Theorem 2.1. of Chan and Lai (2006) could be also applied to obtain a high-level exceedance probability for a maximum of standardized increments of a Brownian bridge, i.e., the limit variable of our test statistic in one-dimensional case. We would like to thank the referee to bring our attention to the paper by Chan and Lai (2006).

Our paper shows how the new probabilistic results concerning Gaussian fields may be applied in statistics. To show how accurate the approximations are, we included a small simulation study where we compare critical values obtained by simulation with critical values obtained by the suggested approximations.

\section{Mathematical formulation of problem}

\section{One-dimensional case}

Suppose that we observe a sequence of $d$-dimensional random vectors $\boldsymbol{X}_{1}, \ldots, \boldsymbol{X}_{n}$. We would like to test the null hypothesis $H_{01}$ against the alternative $A_{1}$ :

$$
\begin{aligned}
H_{01}: \boldsymbol{X}_{i}=\boldsymbol{\mu}+\boldsymbol{e}_{i}, & i=1, \ldots, n, \\
A_{1}: \exists \quad 0 \leq k_{1}^{*}<k_{2}^{*} \leq n & \text { satisfying } \quad \alpha n \leq k_{2}^{*}-k_{1}^{*} \leq(1-\beta) n \\
\text { such that } & \\
\boldsymbol{X}_{i}=\boldsymbol{\mu}+\boldsymbol{e}_{i}, & i=1, \ldots, k_{1}^{*} ; \\
\boldsymbol{X}_{i}=\boldsymbol{\mu}+\boldsymbol{\delta}+\boldsymbol{e}_{i}, & i=k_{1}^{*}+1, \ldots, k_{2}^{*} ; \\
\boldsymbol{X}_{i}=\boldsymbol{\mu}+\boldsymbol{e}_{i}, & i=k_{2}^{*}+1, \ldots, n .
\end{aligned}
$$

We suppose that $\boldsymbol{\mu}$ and $\boldsymbol{\delta} \neq \mathbf{0}$ are unknown real vectors. The error terms $\left\{\boldsymbol{e}_{i}\right\}$ are i.i.d. and $E \boldsymbol{e}_{i}=\mathbf{0}, \operatorname{Var} \boldsymbol{e}_{i}=\boldsymbol{\Sigma}$ (known) and $E\left\|\boldsymbol{e}_{i}\right\|^{2+\Delta}<\infty$. The problem was introduced by Levin and Kline (1985) and studied in a general setting by Antoch and Hušková (1996). 
For $0 \leq k_{1}<k_{2} \leq n$ we introduce

$$
\boldsymbol{S}\left(k_{1}, k_{2}\right)=\sum_{i=k_{1}+1}^{k_{2}}\left(\boldsymbol{X}_{i}-\overline{\boldsymbol{X}}\right)=\sum_{i=1}^{k_{2}}\left(\boldsymbol{X}_{i}-\overline{\boldsymbol{X}}\right)-\sum_{i=1}^{k_{1}}\left(\boldsymbol{X}_{i}-\overline{\boldsymbol{X}}\right),
$$

where $\overline{\boldsymbol{X}}$ denotes an average of all observed vectors. The test statistic has a form:

$$
T_{1}^{2}=\max _{\substack{0 \leq k_{1}<k_{2} \leq n \\[\alpha n] \leq k_{2}-k_{1} \leq[(1-\beta) n]}} \frac{\left(\boldsymbol{S}\left(k_{1}, k_{2}\right)\right)^{T} \boldsymbol{\Sigma}^{-1} \boldsymbol{S}\left(k_{1}, k_{2}\right)}{\left(k_{2}-k_{1}\right)\left(1-\left(k_{2}-k_{1}\right) / n\right)}
$$

For $l=1, \ldots, d$ we introduce zero mean unit variance Gaussian fields

$$
\left\{Y_{l}\left(x_{1}, x_{2}\right)=\frac{B_{l}\left(x_{2}\right)-B_{l}\left(x_{1}\right)}{\sqrt{\left(x_{2}-x_{1}\right)\left(1-\left(x_{2}-x_{1}\right)\right)}}, 0<x_{1}<x_{2}<1\right\},
$$

where $\left\{\left(B_{1}(x), \ldots, B_{d}(x)\right)^{T}, 0 \leq x \leq 1\right\}$ is a $d$-dimensional Gaussian process whose coordinates are independent Brownian bridges.

Then, under $H_{0}$ it holds:

$$
T_{1}^{2} \stackrel{D}{\rightarrow} \max _{\substack{0 \leq x_{1}<x_{2} \leq 1 \\ \alpha \leq x_{2}-x_{1} \leq(1-\beta)}} \sum_{l=1}^{d}\left(Y_{l}\left(x_{1}, x_{2}\right)\right)^{2} \quad \text { as } n \rightarrow \infty .
$$

The assertion (3) may be proved using Theorem 3 of Bickel and Wichura (1971).

The exact distribution of (2) is unknown. In one-dimensional case Yao (1993) derived an accurate tail approximation for normally distributed random variables $\left\{X_{i}\right\}$. The exact distribution of the limit variable given by the righthand side of (3) is also unknown but it is again possible to derive its "tail behaviour".

In what follows the sign " " means an asymptotic equivalence.

Theorem 1. Under the above set up

$P\left(\max _{\substack{0 \leq x_{1}<x_{2} \leq 1 \\ \alpha \leq x_{2}-x_{1} \leq(1-\beta)}} \sum_{l=1}^{d}\left(Y_{l}\left(x_{1}, x_{2}\right)\right)^{2}>u^{2}\right) \sim \frac{1}{2^{d / 2-1} \Gamma(d / 2)} C_{1}(\alpha, \beta) u^{2+d} e^{-u^{2} / 2}$ 
as $u \rightarrow \infty$, where

$$
C_{1}(\alpha, \beta)=\frac{1}{4}\left(\log \frac{(1-\beta)(1-\alpha)}{\beta \alpha}+\frac{1}{\alpha}-\frac{1}{1-\beta}\right)
$$

Proof. We introduce a locally homogeneous Gaussian field

$$
\begin{aligned}
& \left\{Y P\left(x_{1}, x_{2}, \theta_{1}, \ldots, \theta_{d-1}\right), 0<x_{1}<x_{2}<1,-\frac{\pi}{2} \leq \theta_{k} \leq \frac{\pi}{2}, k=1, \ldots, d-2,\right. \\
& \left.-\pi \leq \theta_{d-1} \leq \pi\right\} \quad \text { by } \\
& Y P\left(x_{1}, x_{2}, \theta_{1}, \ldots, \theta_{d-1}\right)=Y_{1}\left(x_{1}, x_{2}\right) u_{1}\left(\theta_{1}, \ldots, \theta_{d-1}\right)+\ldots \\
& \quad+Y_{d}\left(x_{1}, x_{2}\right) u_{d}\left(\theta_{1}, \ldots, \theta_{d-1}\right),
\end{aligned}
$$

where $u_{1}\left(\theta_{1}, \ldots, \theta_{d-1}\right)=\sin \theta_{1}, \ldots, u_{d}\left(\theta_{1}, \ldots, \theta_{d-1}\right)=\cos \theta_{d-1} \cdots \cos \theta_{1}$ are spherical coordinates. Clearly,

$$
\max _{\substack{0 \leq x_{1}<x_{2} \leq 1 \\ \alpha \leq x_{2}-x_{1} \leq(1-\beta)}} \sqrt{\sum_{l=1}^{d}\left(Y_{l}\left(x_{1}, x_{2}\right)\right)^{2}}=\max _{Q} Y P\left(x_{1}, x_{2}, \theta_{1}, \ldots, \theta_{d-1}\right),
$$

where $Q=\left\{\left(x_{1}, x_{2}, \theta_{1}, \ldots, \theta_{d-1}\right), 0<x_{1}<x_{2}<1, \alpha \leq x_{2}-x_{1} \leq(1-\right.$ $\left.\beta),-\frac{\pi}{2} \leq \theta_{k} \leq \frac{\pi}{2}, k=1, \ldots, d-2,-\pi \leq \theta_{d-1} \leq \pi\right\}$. The correlation function of $\left\{Y P\left(x_{1}, x_{2}, \theta_{1}, \ldots, \theta_{d-1}\right)\right\}$ satisfies

$E\left(Y P\left(x_{1}+h_{1}, x_{2}+h_{2}, \theta_{1}+\psi_{1}, \ldots, \theta_{d-1}+\psi_{d-1}\right) Y P\left(x_{1}, x_{2}, \theta_{1}, \ldots, \theta_{d-1}\right)\right)=$ $1-\frac{1}{2\left(x_{2}-x_{1}\right)\left(1-\left(x_{2}-x_{1}\right)\right)}\left|h_{1}\right|-\frac{1}{2\left(x_{2}-x_{1}\right)\left(1-\left(x_{2}-x_{1}\right)\right)}\left|h_{2}\right|-$ $-\frac{1}{2} \psi_{1}^{2}-\frac{1}{2}\left(\cos ^{2} \theta_{1}\right) \psi_{2}^{2}-\cdots-\frac{1}{2}\left(\cos ^{2} \theta_{1} \cdots \cos ^{2} \theta_{d-2}\right) \psi_{d-1}^{2}+o\left(\left|h_{1}\right|+\left|h_{2}\right|+\right.$ $\left.+\psi_{1}^{2}+\cdots+\psi_{d-1}^{2}\right)$ as $h_{1} \rightarrow 0, h_{2} \rightarrow 0, \psi_{1} \rightarrow 0, \ldots, \psi_{d-1} \rightarrow 0$.

Using Theorem 7.1 (see also Corollary 7.5) of Piterbarg (1996) it holds (as $u \rightarrow \infty)$

$$
\begin{aligned}
& P\left(\max _{Q} Y P\left(x_{1}, x_{2}, \theta_{1}, \ldots, \theta_{d-1}\right)>u\right) \sim H_{1,1,2, \ldots, 2} u^{2+d} \frac{1}{\sqrt{2 \pi}} e^{-u^{2} / 2} \times \\
& \int_{Q} \frac{1}{4\left(x_{2}-x_{1}\right)^{2}\left(1-\left(x_{2}-x_{1}\right)\right)^{2}} \prod_{i=1}^{d-2} \sqrt{\frac{1}{2} \cos ^{2 i} \theta_{d-1-i}} d \theta_{1} \ldots \theta_{d-1} d x_{1} d x_{2} .
\end{aligned}
$$


We get (4) as

$$
\begin{gathered}
H_{1,1,2, \ldots, 2}=1 / \pi^{(d-1) / 2} \\
C_{1}(\alpha, \beta)=\iint_{\substack{\alpha \leq x_{2}-x_{1} \leq(1-\beta) \\
0 . x_{2} \leq 1}} \frac{1}{4\left(x_{2}-x_{1}\right)^{2}\left(1-\left(x_{2}-x_{1}\right)^{2}\right.} d x_{1} d x_{2} \\
=\int_{\alpha}^{1-\beta} \int_{0}^{1-x} \frac{1}{4 x^{2}(1-x)^{2}} d \xi d x=\int_{\alpha}^{1-\beta} \frac{1}{4 x^{2}(1-x)} d x \\
=\frac{1}{4}\left(\log \frac{(1-\beta)(1-\alpha)}{\beta \alpha}+\frac{1}{\alpha}-\frac{1}{1-\beta}\right)
\end{gathered}
$$

and

$$
\frac{1}{2^{(d-1) / 2}} \int_{-\pi}^{\pi} \ldots \int_{-\pi / 2}^{\pi / 2} \prod_{i=1}^{d-2} \cos ^{i} \theta_{d-1-i} d \theta_{1} \ldots d \theta_{d-1}=\frac{1}{2^{(d-1) / 2}} \frac{2 \pi^{d / 2}}{\Gamma(d / 2)} .
$$

For $n$ large the approximation (4) may serve to find approximate critical values of the test statistic (2).

Remark 1. In case the variance-covariance matrix $\Sigma$ is unknown, it can be replaced by any consistent estimator.

Remark 2. For $d=1$ we can also consider a one-sided alternative (e.g. $\delta>0)$. The test statistic

$$
\widetilde{T}_{1}=\max _{\substack{0 \leq k_{1}<k_{2} \leq n \\[\alpha n] \leq k_{2}-k_{1} \leq[(1-\beta) n]}} \frac{S\left(k_{1}, k_{2}\right)}{\sigma \sqrt{\left(k_{2}-k_{1}\right)\left(1-\left(k_{2}-k_{1}\right) / n\right)}}
$$

converges $($ as $n \rightarrow \infty)$ in distribution to

$$
\max _{\substack{0 \leq x_{1}<x_{2} \leq 1 \\ \alpha \leq x_{2}-x_{1} \leq(1-\beta)}} \frac{B_{1}\left(x_{2}\right)-B_{1}\left(x_{1}\right)}{\sqrt{\left(x_{2}-x_{1}\right)\left(1-\left(x_{2}-x-1\right)\right)}} .
$$


To find approximate critical values we may apply the approximation (valid for $u$ large) that follows from the limit behaviour as $y \rightarrow \infty$ :

$$
P\left(\max _{\substack{0 \leq x_{1}<x_{2} \leq 1 \\ \alpha \leq x_{2}-x_{1} \leq(1-\beta)}} \frac{B_{1}\left(x_{2}\right)-B_{1}\left(x_{1}\right)}{\sqrt{\left(x_{2}-x_{1}\right)\left(1-\left(x_{2}-x-1\right)\right)}}>u\right) \sim C_{1}(\alpha, \beta) u^{3} \phi(u),
$$

where $\phi(u)$ is the density of a standard normal distribution. As it has been already mentioned, (7) can be also obtained using Theorem 2.1 and Lemma 2.3 of Chan and Lai (2006).

Remark 3. We can consider a more general alternative supposing that a shift in mean may occur not only in one interval but in a union of a fixed number of intervals. For such a case a test statistic as well as its limit behaviour that enables to calculate approximate critical values can be derived analogously. However, with an increase number of change points the convergence to a limit distribution will be slower and approximate critical values less accurate.

\section{Two - dimensional case}

Suppose that on two-dimensional grid $\{(i, j), i=1, \ldots, n ; j=1, \ldots, n\}$ we observe a $d$-dimensional array $\left\{\boldsymbol{X}_{i j}, i=1, \ldots, n, j=1, \ldots, n\right\}$. We would like to test the following hypotheses testing problem

$$
\begin{aligned}
& H_{02}: \boldsymbol{X}_{i j}=\boldsymbol{\mu}+\boldsymbol{e}_{i j}, \quad i=1, \ldots, n, j=1, \ldots, n \\
& A_{2}: \exists \quad 0 \leq k_{1}^{*}<k_{2}^{*} \leq n \text { and } 0 \leq m_{1}^{*}<m_{2}^{*}<n \\
& \text { satisfying } \alpha n^{2} \leq\left(k_{2}^{*}-k_{1}^{*}\right)\left(m_{2}^{*}-m_{1}^{*}\right) \leq(1-\beta) n^{2} \quad \text { such that } \\
& \boldsymbol{X}_{i j}=\boldsymbol{\mu}+\boldsymbol{e}_{i j}, \quad(i, j) \notin\left[k_{1}^{*}, k_{2}^{*}\right] \times\left[m_{1}^{*}, m_{2}^{*}\right], \\
& \boldsymbol{X}_{i j}=\boldsymbol{\mu}+\boldsymbol{\delta}+\boldsymbol{e}_{i j}, \quad(i, j) \in\left[k_{1}^{*}, k_{2}^{*}\right] \times\left[m_{1}^{*}, m_{2}^{*}\right] .
\end{aligned}
$$

We suppose that $\boldsymbol{\mu}$ and $\boldsymbol{\delta} \neq \mathbf{0}$ are unknown real vectors. The error terms $\left\{\boldsymbol{e}_{i j}\right\}$ are i.i.d. and $E \boldsymbol{e}_{i j}=\mathbf{0}, \operatorname{Var} \boldsymbol{e}_{i j}=\boldsymbol{\Sigma}$ (known) and $E\left\|\boldsymbol{e}_{i j}\right\|^{2+\Delta}<\infty$.

Denote $\boldsymbol{S}\left(k_{1}, m_{1}, k_{2}, m_{2}\right)=\sum_{i=k_{1}}^{k_{2}} \sum_{j=m_{1}}^{m_{2}}\left(\boldsymbol{X}_{i j}-\overline{\boldsymbol{X}}\right)$ where 
$\overline{\boldsymbol{X}}=\frac{1}{n^{2}} \sum_{i=1}^{n} \sum_{j=1}^{n} \boldsymbol{X}_{i j}$. The test statistic has a form

$$
T_{2}^{2}=\max _{\substack{0 \leq k_{1}<k_{2} \leq n ; 0 \leq m_{1}<m_{2} \leq n \\\left[\alpha n^{2}\right] \leq\left(m_{2}-m_{1}\right)\left(k_{2}-k_{1}\right) \leq\left[(1-\beta) n^{2}\right]}} \frac{\frac{1}{n}\left(\boldsymbol{S}\left(k_{1}, m_{1}, k_{2}, m_{2}\right)\right)^{T} \boldsymbol{\Sigma}^{-1} \boldsymbol{S}\left(k_{1}, k_{2}, m_{1}, m_{2}\right)}{\sqrt{\frac{\left(k_{2}-k_{1}\right)}{n} \frac{\left(m_{2}-m_{1}\right)}{n}\left(1-\frac{\left(k_{2}-k_{1}\right)\left(m_{2}-m_{1}\right)}{n^{2}}\right)}} .
$$

For $l=1, \ldots, d$ we introduce zero mean Gaussian fields $\left\{Z_{l}\left(x_{1}, y_{1}, x_{2}, y_{2}\right)\right.$, $\left.0<x_{1}<x_{2}<1,0<y_{1}<y_{2}<1\right\}$ defined by

$$
\begin{aligned}
& Z_{l}\left(x_{1}, y_{1}, x_{2}, y_{2}\right)= \\
& \frac{W_{l}\left(x_{2}, y_{2}\right)-W_{l}\left(x_{2}, y_{1}\right)-W_{l}\left(x_{1}, y_{2}\right)+W_{l}\left(x_{1}, y_{1}\right)-\left(x_{2}-x_{1}\right)\left(y_{2}-y_{1}\right) W_{l}(1,1)}{\sqrt{\left(x_{2}-x_{1}\right)\left(y_{2}-y_{1}\right)\left(1-\left(x_{2}-x_{1}\right)\left(y_{2}-y_{1}\right)\right)}}
\end{aligned}
$$

where $\left\{\left(W_{1}(x, y), \ldots, W_{d}(x, y)\right)^{T}, x \geq 0, y \geq 0\right\}$ is a zero mean Gaussian field whose coordinates are independent Gaussian fields with a covariance structure

$$
E\left(W_{l}\left(x_{1}, y_{1}\right) W_{l}\left(x_{2}, y_{2}\right)\right)=\min \left(x_{1}, x_{2}\right) \min \left(y_{1}, y_{2}\right), \quad l=1, \ldots, d .
$$

We also introduce a subset $A$ of $R^{4}$ by $A=\left\{0 \leq x_{1}<x_{2} \leq 1,0 \leq y_{1}<y_{2} \leq\right.$ $\left.1, \alpha \leq\left(x_{2}-x_{1}\right)\left(y_{2}-y_{1}\right) \leq(1-\beta)\right\}$. Applying again results by Bickel and Wichura (1971) we get

$$
T_{2}^{2} \stackrel{D}{\rightarrow} \max _{\left(x_{1}, y_{1}, x_{2}, y_{2}\right) \in A} \sum_{l=1}^{d}\left(Z_{l}\left(x_{1}, y_{1}, x_{2}, y_{2}\right)\right)^{2} \quad \text { as } \quad n \rightarrow \infty .
$$

Theorem 2. Under the above set up

$$
\begin{aligned}
& P\left(\max _{\left(x_{1}, y_{1}, x_{2}, y_{2}\right) \in A} \sum_{l=1}^{d}\left(Z_{l}\left(x_{1}, y_{1}, x_{2}, y_{2}\right)\right)^{2}>u^{2}\right) \\
& C_{2}(\alpha, \beta) \frac{1}{2^{d / 2-1} \Gamma(d / 2)} u^{6+d} e^{-u^{2} / 2},
\end{aligned}
$$

as $u \rightarrow \infty$, where

$$
C_{2}(\alpha, \beta)=\int_{\alpha}^{1-\beta} \frac{-2(1-\xi)-(1+\xi) \log \xi}{16 \xi^{2}(1-\xi)^{4}} d \xi .
$$


Proof. The zero mean Gaussian field

$$
\begin{aligned}
& Z P\left(x_{1}, y_{1}, x_{2}, y_{2}, \theta_{1}, \ldots, \theta_{d-1}\right)=Z_{1}\left(x_{1}, y_{1}, x_{2}, y_{2}\right) u_{1}\left(\theta_{1}, \ldots, \theta_{d-1}\right)+ \\
&+ Z_{d}\left(x_{1}, y_{1}, x_{2}, y_{2}\right) u_{d}\left(\theta_{1}, \ldots, \theta_{d-1}\right)
\end{aligned}
$$

is locally homogeneous. Denote $x=x_{2}-x_{1}, y=y_{2}-y_{1}$. The correlation function of $\left\{Z P\left(x_{1}, y_{1}, x_{2}, y_{2}, \theta_{1}, \ldots, \theta_{d-1}\right)\right\}$ satisfies

$$
\begin{aligned}
& E\left(Z P\left(x_{1}+h_{1}, y_{1}+f_{1}, x_{2}+h_{2}, y_{2}+f_{2}, \theta_{1}+\psi_{1}, \ldots, \theta_{d-1}+\psi_{d-1}\right)\right. \\
& \left.Z P\left(x_{1}, y_{1}, x_{2}, y_{2}, \theta_{1}, \ldots, \theta_{d-1}\right)\right)= \\
& 1-\frac{1}{2 x(1-x y)}\left|h_{1}\right|-\frac{1}{2 x(1-x y)}\left|h_{2}\right|--\frac{1}{2 y(1-x y)}\left|f_{1}\right|-\frac{1}{2 y(1-x y)}\left|f_{2}\right|- \\
& -\frac{1}{2} \psi_{1}^{2}-\frac{1}{2}\left(\cos ^{2} \theta_{1}\right) \psi_{2}^{2}-\cdots-\frac{1}{2}\left(\cos ^{2} \theta_{1} \cdots \cos ^{2} \theta_{d-1}\right) \psi_{d-1}^{2}+ \\
& +o\left(\left|h_{1}\right|+\left|h_{2}\right|+\left|f_{1}\right|+\left|f_{2}\right|+\psi_{1}^{2}+\cdots+\psi_{d-1}^{2}\right)
\end{aligned}
$$

as $h_{1} \rightarrow 0, h_{2} \rightarrow 0, f_{1} \rightarrow 0, f_{2} \rightarrow 0, \psi_{1} \rightarrow 0, \ldots, \psi_{d-1} \rightarrow 0$. The assertion follows again from Theorem 7.1 of Piterbarg (1996), where

$$
\begin{aligned}
& C_{2}(\alpha, \beta)= \\
& \iiint \int_{A} \frac{1}{16\left(x_{2}-x_{1}\right)^{2}\left(y_{2}-y_{1}\right)^{2}\left(1-\left(y_{2}-y_{1}\right)\left(x_{2}-x_{1}\right)\right)^{4}} d x_{1} d x_{2} d y_{1} d y_{2} \\
& =\iint_{\alpha \leq x} \frac{(1-x)(1-y)}{16 x^{2} y^{2}(1-x y)^{4}} d x d y \\
& =\int_{\alpha}^{1-\beta} \frac{1}{16 \xi^{2}(1-\xi)^{4}} d \xi \int_{\xi}^{1} \frac{(\eta-\xi)(1-\eta)}{\eta^{2}} d \eta=(12) .
\end{aligned}
$$

For $n$ large the approximation (11) may serve for finding approximate critical values of the test statistic (9).

Remark 4. In case the variance-covariance matrix $\Sigma$ is unknown it can be replaced by any consistent estimator. 
Remark 5. For $d=1$ we can also consider a one-sided alternative (e.g. $\delta>0)$. The test statistic

$$
\widetilde{T}_{2}=\max _{\substack{0 \leq k_{1}<k_{2} \leq n ; 0 \leq m_{1}<m_{2} \leq n \\\left[\alpha n^{2}\right] \leq\left(m_{2}-m_{1}\right)\left(k_{2}-k_{1}\right) \leq\left[(1-\beta) n^{2}\right]}} \frac{\frac{1}{\sigma} \frac{1}{n} S\left(k_{1}, m_{1}, k_{2}, m_{2}\right)}{\sqrt{\frac{\left(k_{2}-k_{1}\right)}{n} \frac{\left(m_{2}-m_{1}\right)}{n}\left(1-\frac{\left(k_{2}-k_{1}\right)\left(m_{2}-m_{1}\right)}{n^{2}}\right)}},
$$

converges as $(n \rightarrow \infty)$ in distribution to

$$
\max _{\left(x_{1}, y_{1}, x_{2}, y_{2}\right) \in A} Z_{1}\left(x_{1}, y_{1}, x_{2}, y_{2}\right)
$$

and

$$
P\left(\max _{\left(x_{1}, y_{1}, x_{2}, y_{2}\right) \in A} Z_{1}\left(x_{1}, y_{1}, x_{2}, y_{2}\right)>u\right) \sim C_{2}(\alpha, \beta) u^{7} \phi(u) \quad \text { as } \quad u \rightarrow \infty .
$$

The limit behaviour (14) may be also obtained as a consequence of Theorem 2.1 and Lemma 2.3 of Chan and Lai (2006).

Remark 6. We can again consider a more general alternative supposing that a shift in mean can occur on a more complex set $A$. For applications a limit distribution of a corresponding test statistic, if the set $A$ has a form of an ellipsoid, may be of interest. This may be a goal for our future research.

\section{Transient change in a parameter of a distribution}

The problem (1) can be called a test for an "epidemic type of change" in mean. However, it can be formulated in more general way as a transient change in a multidimensional parameter $\boldsymbol{\theta}$ of a distribution. More precisely, we suppose that $\boldsymbol{X}_{1}, \ldots, \boldsymbol{X}_{n}$ are independent vectors distributed according to distributions with densities $f\left(\boldsymbol{x}_{1} ; \boldsymbol{\theta}_{1}, \boldsymbol{\eta}_{1}\right), \ldots, f\left(\boldsymbol{x}_{n} ; \boldsymbol{\theta}_{n}, \boldsymbol{\eta}_{n}\right)$ where $\boldsymbol{\theta}_{1}, \ldots, \boldsymbol{\theta}_{n} \in \Theta^{(1)} \subset R^{d}$ and $\boldsymbol{\eta}_{1}, \ldots, \boldsymbol{\eta}_{n} \in \Theta^{(2)} \subset R^{c}$ are parameters. We would like to test the null hypothesis $H_{03}$ against the alternative $A_{3}$ : 


$$
\begin{aligned}
& H_{03}: \boldsymbol{\theta}_{1}=\cdots=\boldsymbol{\theta}_{n} \text { and } \boldsymbol{\eta}_{1}=\cdots=\boldsymbol{\eta}_{n}=\boldsymbol{\eta} \\
& A_{3}: \exists \quad 0 \leq k_{1}^{*}<k_{2}^{*} \leq n \quad \text { satisfying } \quad \alpha n \leq k_{2}^{*}-k_{1}^{*} \leq(1-\beta) n \\
& \text { such that } \\
& \quad \boldsymbol{\theta}_{1}=\cdots=\boldsymbol{\theta}_{k_{1}^{*}} \neq \boldsymbol{\theta}_{k_{1}^{*}+1}=\cdots=\boldsymbol{\theta}_{k_{2}^{*}} \neq \boldsymbol{\theta}_{k_{2}^{*}+1}=\cdots=\boldsymbol{\theta}_{n}, \\
& \boldsymbol{\theta}_{1}=\cdots=\boldsymbol{\theta}_{k_{1}^{*}}=\boldsymbol{\theta}_{k_{2}^{*}+1}=\cdots=\boldsymbol{\theta}_{n}, \\
& \boldsymbol{\eta}_{1}=\cdots=\boldsymbol{\eta}_{n}=\boldsymbol{\eta},
\end{aligned}
$$

where $\boldsymbol{\theta}_{1}, \ldots, \boldsymbol{\theta}_{n}$ are parameters of interest while $\boldsymbol{\eta}_{\mathbf{1}}, \ldots, \boldsymbol{\eta}_{\boldsymbol{n}}$ are nuisance parameters, see Csörgő and Horváth (1997). If we consider the log-likelihood ratio test statistic in the form

$$
L^{2}=\max _{\substack{0 \leq k_{1}<k_{2} \leq n \\[\alpha n] \leq k_{2}-k_{1} \leq[(1-\beta) n]}}-2 \log \Lambda_{k_{1}, k_{2}}
$$

with

$$
\Lambda_{k_{1}, k_{2}}=\frac{\sup _{\boldsymbol{\theta}, \boldsymbol{\eta}} \prod_{1 \leq i \leq n} f\left(\boldsymbol{X}_{i} ; \boldsymbol{\theta}, \boldsymbol{\eta}\right)}{\sup _{\boldsymbol{\theta}_{1}, \boldsymbol{\theta}_{2}, \boldsymbol{\eta}} \prod_{\substack{1 \leq i \leq k_{1} \\ k_{2}<i \leq n}} f\left(\boldsymbol{X}_{i} ; \boldsymbol{\theta}_{1}, \boldsymbol{\eta}\right) \prod_{k_{1}<i \leq k_{2}} f\left(\boldsymbol{X}_{i} ; \boldsymbol{\theta}_{2}, \boldsymbol{\eta}\right)},
$$

then under the condition C.1 - C.9 (or their analogues) of Csörgö and Horváth (1997) we get again

$$
L^{2} \stackrel{D}{\rightarrow} \max _{\substack{0 \leq x_{1}<x_{2} \leq 1 \\ \alpha \leq x_{2}-x_{1} \leq(1-\beta)}} \sum_{l=1}^{d}\left(Y_{l}\left(x_{1}, x_{2}\right)\right)^{2} \quad \text { as } \quad n \rightarrow \infty .
$$

For instance, if we test for a transient change in the one-dimensional parameter $\theta$ of an exponential distribution (two-sided alternative) the test statistic (16) is equivalent to the test statistic

$$
\left|T_{e}\right|=\max _{\substack{0 \leq k_{1}<k_{2} \leq n \\[\alpha n] \leq k_{2}-k_{1} \leq[(1-\beta) n]}} \frac{\left|S\left(k_{1}, k_{2}\right)\right|}{\bar{X} \sqrt{\left(k_{2}-k_{1}\right)\left(1-\left(k_{2}-k_{1}\right) / n\right)}} .
$$

For one-sided alternative, the test statistic has a form

$$
\widetilde{T}_{e}=\max _{\substack{0 \leq k_{1}<k_{2} \leq n \\[\alpha n] \leq k_{2}-k_{1} \leq[(1-\beta) n]}} \frac{S\left(k_{1}, k_{2}\right)}{\bar{X} \sqrt{\left(k_{2}-k_{1}\right)\left(1-\left(k_{2}-k_{1}\right) / n\right)}} .
$$


The test statistic (18), resp. (19), may be applied for testing a transient change in an intensity $\lambda$ of a one-dimensional Poisson process because intervals between successive events have an exponential distribution. Instead of (18) we may use for a two-sided alternative a test statistic

$$
\left|P_{1}\right|=\max _{\substack{0 \leq t_{1}<t_{2} \leq T \\ \alpha \leq t_{2}-t_{1} \leq(1-\beta) T}} \frac{\left|N\left(\left(t_{1}, t_{2}\right)\right)-\left(t_{2}-t_{1}\right) N((0, T)) / T\right|}{\sqrt{N((0, T)) \frac{t_{2}-t_{1}}{T}\left(1-\frac{t_{2}-t_{1}}{T}\right)}}
$$

and for a one-sided alternative a test statistic

$$
\widetilde{P}_{1}=\max _{\substack{0 \leq t_{1}<t_{2} \leq T \\ \alpha T \leq t_{2}-t_{1} \leq(1-\beta) T}} \frac{N\left(\left(t_{1}, t_{2}\right)\right)-\left(t_{2}-t_{1}\right) N((0, T)) / T}{\sqrt{N((0, T)) \frac{t_{2}-t_{1}}{T}\left(1-\frac{t_{2}-t_{1}}{T}\right)}},
$$

where $N(A)$ denotes a number of events in a set $A$. Under the null hypothesis of no change in an intensity it holds (by applying Bickel and Wichura (1971))

$$
\left|P_{1}\right| \stackrel{D}{\rightarrow} \max _{\substack{0 \leq x_{1}<x_{2} \leq 1 \\ \alpha \leq x_{2}-x_{1} \leq(1-\beta)}}\left|Y_{1}\left(x_{1}, x_{2}\right)\right|, \quad \widetilde{P}_{1} \stackrel{D}{\rightarrow} \max _{\substack{0 \leq x_{1}<x_{2} \leq 1 \\ \alpha \leq x_{2}-x_{1} \leq(1-\beta)}} Y_{1}\left(x_{1}, x_{2}\right)
$$

as $T \rightarrow \infty$.

The same asymptotic results were obtained by Loader (1991) together with a more accurate approximation (for smaller value of $T$ ) based on large deviations theory. The analogue test statistics may be derived for testing a transient change in an intensity of a Poisson process in a plane, more precisely for a two-sided alternative

$$
\left|P_{2}\right|=\max _{\substack{1 \leq t_{1}<t_{2} \leq T \\ 1 \leq s_{1}<s_{2} \leq T \\ \alpha T^{2} \leq\left(t_{2}-t_{1}\right)\left(s_{2}-s_{1}\right) \leq(1-\beta) T^{2}}} \frac{\left|N\left(\left[t_{1}, t_{2}\right] \times\left[s_{1}, s_{2}\right]\right)-\frac{\left(t_{2}-t_{1}\right)\left(s_{2}-s_{1}\right)}{T^{2}} N\left([0, T]^{2}\right)\right|}{\sqrt{N\left([0, T]^{2}\right) \frac{\left(t_{2}-t_{1}\right)\left(s_{2}-s_{1}\right)}{T^{2}}\left(1-\frac{\left(t_{2}-t_{1}\right)\left(s_{2}-s_{1}\right)}{T^{2}}\right)}},
$$

resp. for a one-sided alternative

$$
\widetilde{P}_{2}=\max _{\substack{1 \leq t_{1}<t_{2} \leq T \\ 1 \leq s_{1}<s_{2} \leq T \\ \alpha T^{2} \leq\left(t_{2}-t_{1}\right)\left(s_{2}-s_{1}\right) \leq(1-\beta) T^{2}}} \frac{N\left(\left[t_{1}, t_{2}\right] \times\left[s_{1}, s_{2}\right]\right)-\frac{\left(t_{2}-t_{1}\right)\left(s_{2}-s_{1}\right)}{T^{2}} N\left([0, T]^{2}\right)}{\sqrt{N\left([0, T]^{2}\right) \frac{\left(t_{2}-t_{1}\right)\left(s_{2}-s_{1}\right)}{T^{2}}\left(1-\frac{\left(t_{2}-t_{1}\right)\left(s_{2}-s_{1}\right)}{T^{2}}\right)}} .
$$


Under the null hypothesis of no change in a intensity it holds

$$
\left|P_{2}\right| \stackrel{D}{\rightarrow} \max _{\left(x_{1}, y_{1}, x_{2}, y_{2}\right) \in A}\left|Z_{1}\left(x_{1}, y_{1}, x_{2}, y_{2}\right)\right|,
$$

and

$$
\widetilde{P}_{2} \stackrel{D}{\rightarrow} \max _{\left(x_{1}, y_{1}, x_{2}, y_{2}\right) \in A} Z_{1}\left(x_{1}, y_{1}, x_{2}, y_{2}\right)
$$

as $T \rightarrow \infty$.

The results (25) and (26) were again obtained by Loader (1991) together with a better approximation for smaller value of $T$.

\section{Simulation study}

The quality of the suggested approximations is affected by distribution (under $H_{0}$ ) of observations, and by a choice of $\alpha$ and $\beta$. The agreement is worse for smaller value of $\alpha$, resp. $\beta$. For comparison Table 1 and 2 present the $5 \%$ critical values of $\widetilde{T}_{1}$ and $\widetilde{T}_{e}$, (i.e. $d=1$ and one sided-alternative) for normally and exponential distributed variables for several choice of $\alpha$ and $\beta$, obtained by (7) and by simulations.

\begin{tabular}{|c|c|c|c|c|}
\hline trimmed portions & $(7)$ & $n=1000$ & $n=2000$ & $n=4000$ \\
\hline$\alpha=0.01, \beta=0.01$ & 4.44 & 4.13 & 4.24 & 4.27 \\
\hline$\alpha=0.20, \beta=0.20$ & 3.57 & 3.46 & 3.50 & 3.51 \\
\hline$\alpha=0.01, \beta=0.95$ & 4.02 & 3.85 & 3.89 & 3.92 \\
\hline
\end{tabular}

Table 1 . The $5 \%$ critical values of $\widetilde{T}_{1}$ for normally distributed observations.

\begin{tabular}{|c|c|c|c|c|}
\hline trimmed portions & $(7)$ & $n=1000$ & $n=2000$ & $n=4000$ \\
\hline$\alpha=0.01, \beta=0.01$ & 4.44 & 5.20 & 4.96 & 4.84 \\
\hline$\alpha=0.20, \beta=0.20$ & 3.57 & 3.54 & 3.56 & 3.61 \\
\hline$\alpha=0.01, \beta=0.95$ & 4.02 & 5.14 & 4.94 & 4.80 \\
\hline
\end{tabular}

Table 2 . The $5 \%$ critical values of $\widetilde{T}_{e}$ for exponentially distributed observations.

Table 3 shows the agreement between the $5 \%$ critical values of $\left|T_{1}\right|$ obtained by (4) and by simulations with normally distributed random vectors for $d=$ $1,2,3$ and $\alpha=\beta=0.01$. 


\begin{tabular}{|c|c|c|c|c|}
\hline trimmed portions & $(4)$ & $n=1000$ & $n=2000$ & $n=4000$ \\
\hline$d=1$ & 4.62 & 4.31 & 4.41 & 4.46 \\
\hline$d=2$ & 5.05 & 4.76 & 4.82 & 4.87 \\
\hline$d=3$ & 5.38 & 5.06 & 5.11 & 5.19 \\
\hline
\end{tabular}

Table 3 . The $5 \%$ critical values of $\left|T_{1}\right|$ for normally distributed observations.

Table 4 shows the $5 \%$ critical values of $\widetilde{T}_{2}$ obtained by (14) and by a simulation study when the variables are normally distributed.

\begin{tabular}{|c|c|c|c|}
\hline trimmed portions & $(14)$ & $n^{2}=1600$ & $n^{2}=3600$ \\
\hline$\alpha=0.20, \beta=0.20$ & 4.72 & 4.09 & 4.12 \\
\hline$\alpha=0.01, \beta=0.95$ & 5.80 & 4.67 & 4.81 \\
\hline
\end{tabular}

Table 4 . The $5 \%$ critical values of $\widetilde{T}_{2}$ for normally distributed observations.

All simulated critical values were obtained from 5000 repetitions.

Acknowledgment: We would like to thank our referees for all their recommendations and comments. Especially, we would like to thank one of the referees to pointing us to the paper by Chan and Lai (2006). The work of the first author was supported by grant GAČR 201/09/0755.

\section{References}

[1] Antoch J., Hušková M., 1996. Tests and estimators for epidemic alternatives. Tatra Mountains Math. Publ. 7, 311-329.

[2] Antoch J., Jarušková D., 2007. Testing a homogeneity of stochastic processes. Kybernetika 43, 415-430.

[3] Bickel P. J., Wichura M. J., 1971. Convergence criteria for multiparameter stochastic processes and some applications. The Annals of Mathematical Statistics 42, 1656-1670.

[4] Chan H.P., Lai T.L., 2006. Maxima of asymptotically Gaussian random fields and moderate deviation approximations to boundary crossing probabilities of sums of random variables with multidimensional indices. The Annals of Probability 34, 80-121.

[5] Csörgő M., Horváth L., 1997. Limit Theorems in Change Point Analysis. J. Wiley, New York. 
[6] Glaz J., Naus J., Wallenstein S., 2001. Scan statistics. Springer, New York-Berlin-Heidelberg.

[7] Levin B., Kline J., 1985. The cusum test of homogeneity with an application to spontaneous abortion epidemiology. Statist. in Medicine 4, $469-488$.

[8] Loader C. R., 1991. Large-deviation approximations to the distribution of scan statistics. Adv. Appl. Prob. 23, 751-771.

[9] Piterbarg V.I., 1996. Asymptotic methods in the theory of Gaussian processes and fields. American Mathematical Society. Providence.

[10] Siegmund D., 1988. Approximate tail probabilities for the maxima of some random fields. The Annals of Probability 16, 487-501.

[11] Siegmund D., Yakir B., 2000 Tail probabilities for the null distribution of scanning statistics. Bernoulli 6, 191-213.

[12] Yao Q., 1993. Boundary-crossing probabilities of some random fields related to likelihood ratio tests for epidemic alternatives. J. Appl. Prob. 30, $52-65$. 